\title{
New Approaches for Treatment of Advanced Extranodal NK/T-Cell Lymphoma
}

\author{
Wu Yi', Tianxin Yang ${ }^{2}$, Sisi Lin', Rui Hao', Jin Yu', Ying Wang $\mathbb{D}^{1,3}$, Xiangming Tong ${ }^{2,4}$ \\ 'Phase I Clinical Research Center, Zhejiang Provincial People's Hospital, People's Hospital of Hangzhou Medical College, Hangzhou, People's Republic \\ of China; ${ }^{2}$ Department of Hematology, Zhejiang Provincial People's Hospital, People's Hospital of Hangzhou Medical College, Hangzhou, People's \\ Republic of China; ${ }^{3}$ Clinical Research Institute, Zhejiang Provincial People's Hospital, People's Hospital of Hangzhou Medical College, Hangzhou, \\ People's Republic of China; ${ }^{4}$ The Key Laboratory of Tumor Molecular Diagnosis and Individualized Medicine of Zhejiang Province, Zhejiang Provincial \\ People's Hospital, People's Hospital of Hangzhou Medical College, Hangzhou, People's Republic of China \\ Correspondence: Xiangming Tong, The Key Laboratory of Tumor Molecular Diagnosis and Individualized Medicine of Zhejiang Province, Zhejiang \\ Provincial People's Hospital, People's Hospital of Hangzhou Medical College, Hangzhou, 3100 I4, People's Republic of China, Tel +86-I3758I83497, \\ Email tongxiangmin@163.com
}

\begin{abstract}
Extranodal NK/T cell lymphoma (ENKL) is a rare subtype of lymphoma that shows a poor clinical outcome. The most common sites are the nasal cavity, nasopharynx, paranasal sinuses, tonsils and larynx. Because of P-glycoprotein expression on ENKL cells, ENKL is resistant to anthracycline-based chemotherapy. L-asparaginase-based chemotherapy with or without radiotherapy shows promising outcomes for advanced ENKL, but has limited efficacy in relapsed/refractory ENKL. immune-checkpoint inhibitors, histone deacetylase inhibitors, and monoclonal antibodies are being investigated. In this review, we summarize the new treatments for ENKL.
\end{abstract} Keywords: extranodal NK/T cell lymphoma, pathway, PD-1, immunotherapy

\section{Background}

Extranodal natural killer/T-cell lymphoma (ENKL) is an aggressive lymphoma. It is characterized by multidrug resistance due to the P-glycoprotein. ${ }^{1}$ According to the classification of tumors of hematopoietic and lymphoid tissues set by the World Health Organization in 2017, mature ENKL can be divided into three subtypes: ENKL; nasal type, aggressive NK-cell leukemia; and chronic lymphoproliferative disorders of NK cells. ${ }^{2}$ Moreover, the classification of EBV-positive lymphoproliferative disorders (LPD) includes chronic active EBV infection (CAEBV) of T- and NK-cell type, systemic EBV-positive T-cell lymphoma of childhood, aggressive NK-cell leukemia, extranodal NK/T-cell lymphoma, nasal type, and the new provisional entity primary EBV-positive nodal T/NK-cell lymphoma. ${ }^{3}$

ENKL, nasal type usually occurs in the upper aerodigestive tract and acquires a dismal outcome when extranasal organs (eg, skin, testes) are involved. ENKL, nasal type is more prevalent in Asian countries than in Western countries, and is associated with Epstein-Barr virus (EBV) infection. Several studies have shown that radiotherapy alone can be recommended as first-line treatment in localized stage-I/II disease. ${ }^{4}$ L-asparaginase-based regimens have a certain therapeutic effect in relapsed/refractory ENKL. ${ }^{5,6}$ Some studies have shown that programmed cell death protein 1 (PD-1) blockade can aid treatment of refractory/relapsed ENKL. ${ }^{7,8}$

In this review, we reviewed the chemotherapy, radiotherapy and hematopoietic stem cell transplantation of ENKL, and explored its immunotherapy especially the progress of PD1 therapy for treating ENKL patients.

\section{Conventional Approaches for Extranodal NK/T-Cell Lymphoma Radiotherapy (RT)}

As we knew the conventional anthracycline-containing (CHOP, cyclophosphamide, adriamycin, vincristine, prednisolone, or CHOP-like) approaches are not applicable for ENKL, because of the P-glycoprotein. ${ }^{1}$ RT plays an important role in limited-stage ENKL. The early trial examined the correlation between local recurrence and radiotherapeutic 
parameters. ${ }^{9}$ Thirty-five patients were included in the study and 32 of them had stage I disease. Seventeen patients only received RT treatment, and the remaining administrated a combination of chemotherapy and RT. The median tumor dose was 50 grays (Gy) (range, 22-60 Gy). The complete remission (CR) or a CR/unconfirmed was $80 \%$. The 5-year overall survival (OS) rate, disease-free survival (DFS) rate, and local control probability (LCP) were $47.3 \%, 42.9 \%$, and $65.2 \%$, respectively. The patients who received RT doses 50 Gy achieved favorable local control.

A large retrospective study of patients with early-staged ENKL was conducted in China. Patients received sequence of RT alone, RT alone, RT followed by CT (chemotherapy), or CT followed by RT. The 5-year overall survival (OS) RT alone and RT with or without CT were $69.6 \%, 67.7 \%$ and $33.9 \%$, respectively. Risk-adapted therapy involving RT alone for lowrisk patients and RT consolidated by CT for high-risk patients is a viable, effective strategy for early-stage ENKL. ${ }^{10}$

The main point is RT alone or followed by $\mathrm{CT}$ can be a feasible and effective treatment strategy for early staged ENKL and appropriate RT dose in localized ENKL is more than $50 \mathrm{~Gy}$.

\section{Chemotherapy (CT)}

SMILE (dexamethasone, methotrexate, ifosfamide, L-asparaginase, and etoposide) regime was widely used in advanced and relapsed disease and demonstrated favorable efficacy but the high profile of toxicity. ${ }^{5}$ Another trial showed promising outcomes in Asian ENKL, 56\% of them at the stage III/IV disease. After 2-3 courses of SMILE were administered, the complete remission (CR) rate, partial remission rate (PR) and the overall response (OR) rate were $56 \%$, $22 \%$ and $78 \%$, respectively. The 5 -year overall survival was $50 \%$ and 4 -year disease-free-survival was $64 \%$ after a median follow-up of 31 months. The toxicities included neutropenia, thrombocytopenia and nephrotoxicity. ${ }^{11}$ A retrospective survey revealed that that modified-SMILE regimen followed by RT was superior to CHOP regimen and the respective 2-year survival rates were $60 \%$ and $40 \%$, respectively. ${ }^{12}$

Other regimens such as P-GMOX (pegaspargase, gemcitabine, oxaliplatin), DDGP (dexamethasone, gemcitabine, cisplatin, pegaspargase) and AspaMetDex (L-asparaginase, methotrexate, and dexamethasone) are listed as suggested treatment regimens in the NCCN guidelines. ${ }^{13}$ In China, a total of 35 patients with newly diagnosed advanced or relapsed/refractory ENKL were treated with 2 to 8 cycles of P-GMOX. The ORR was $80.0 \%$ and a CR rate was $51.4 \%$ upon the finished patients. The main toxicities were hematologic toxicity and liver dysfunction. ${ }^{14}$ Another clinical trial compared the safety and efficacy of DDGP and SMILE regimens in advanced ENKL patients. The CR rate and ORR of the DDGP group and SMILE group were $71 \%$ vs $29 \%, 95 \%$ vs $67 \%$. The toxicity of SMILE group showed more serious than the DDGP group. ${ }^{15}$ A Phase II study of AspaMetDex (L-asparaginase, methotrexate, and dexamethasone) was administered in relapsed or refractory ENKL patients. Nineteen patients were enrolled in this study and 11 patients got CR (61\%) after 3 cycles of chemotherapy. The major adverse events were hepatitis, cytopenia, and allergy. ${ }^{6}$ The most common chemotherapy regimens are listed in Table 1.

\section{Hematopoietic Stem Cell Transplantation (HSCT)}

The current view is that autologous HSCT (auto-HSCT) is not recommended in limited-stage ENKL because of a lack of improvement seen in patients with limited-stage disease. ${ }^{16,17}$

The outcomes of auto-HSCT in patients with advanced disease seem comparable with those obtained with L-asparaginase-containing regimens. In a retrospective analysis of 62 patients undergoing auto-HSCT, ${ }^{18}$ at a median follow-up of 43.3 months, the 3-year overall survival (OS) and progression-free survival (PFS) for limited-stage disease were $67.6 \%$ and $64.5 \%$, and those for advanced-stage disease were $52.3 \%$ and $40.1 \%$, respectively. Another retrospective study showed a benefit of auto-HSCT in those with high International Prognostic Index risk scores. ${ }^{16}$ Therefore, autoHSCT as consolidation therapy for advanced ENKL is controversial.

In a retrospective study, in 18 patients with ENKL who underwent allogeneic-HSCT, the 5-year OS was 57\% and 5 -year event-free survival (EFS) was $51 \%$ after a median follow-up of 20.5 months. Use of the SMILE (dexamethasone, methotrexate (MTX), ifosfamide, L-asparaginase, etoposide) regimen pre-HSCT was the most important positive prognostic indicator, resulting in significantly superior OS and EFS $(\mathrm{P}<0.01) .{ }^{19}$

A recent retrospective study analyzed the outcome of 82 ENKL patients undergoing allogeneic-HSCT, with a majority having received peripheral blood grafts $(89 \%)$ from matched related donors $(61 \%) .{ }^{20}$ At a median follow- 
Table I Summary of Chemotherapy Regimens ENKL

\begin{tabular}{|c|c|c|c|c|c|}
\hline Regime & Design & Population & CR\% & OR\% & Toxicity \\
\hline SMILE & Phase II study & $\begin{array}{l}\text { Stage IV, relapsed, or } \\
\text { refractory }\end{array}$ & $45 \%$ ( 2 cycles $)$ & $55 \%$ (I year) & $61 \%$ grade $3-4$ infection \\
\hline P-GMOX & Retrospective & $\begin{array}{l}\text { Stage III-IV, relapsed } \\
\text { or refractory }\end{array}$ & $51.4 \%$ (4 months) & $80.0 \%$ (4 months) & $\begin{array}{l}40 \% \text { grade } 3-4 \text { eucopenia and } \\
\text { neutropenia }\end{array}$ \\
\hline DDGP & RCT & Stage III-IV & $71 \%$ (I year) & 95\% (I year) & $71 \%$ neutropenia \\
\hline AspaMetDex & Phase II study & $\begin{array}{l}\text { Relapsed or } \\
\text { refractory }\end{array}$ & $61 \%$ (I year) & $78 \%$ (I year) & $\begin{array}{l}\text { Grade } 4 \text { neutropenia }(n=1) \\
\text { grade } 3-4 \text { infection }(n=2)\end{array}$ \\
\hline MEDA & Retrospective & $\begin{array}{l}\text { Relapsed or } \\
\text { refractory }\end{array}$ & $61.5 \%$ (4 months) & $76.9 \%$ (4 months) & $\begin{array}{l}\text { 15.4\% Serious infections; } 30.8 \% \text { Grade } \\
3 / 4 \text { thrombocytopenia } \\
23.1 \% \text { Grade } 3 / 4 \text { anemia }\end{array}$ \\
\hline $\begin{array}{l}\text { Sequential } \\
\text { DICE-L-asp }\end{array}$ & Retrospective & $\begin{array}{l}\text { Newly diagnosed, } \\
\text { stage IE to IIE }\end{array}$ & $90.9 \%$ (4 months) & $82 \%$ (5 years) & $\begin{array}{l}\text { 75.76\% Grade } 3 / 4 \text { Leukopenia; } \\
\text { 27.27\% Grade } 3 / 4 \text { Febrile neutropenia } \\
63.64 \% \text { Grade } 3 / 4 \text { Neuropathy }\end{array}$ \\
\hline
\end{tabular}

Abbreviations: SMILE, dexamethasone, methotrexate, ifosfamide, L-asparaginase, and etoposide; P-GemOx, peg-asparaginase, gemcitabine, oxaliplatin; DDGP, dexamethasone, cisplatin, gemcitabine, peg-asparaginase; AspMetDex, asparaginase, methotrexate, dexamethasone; MEDA, high-dose methotrexate, etoposide, dexamethasone, and pegaspargase; Sequential DICE-L-asp, cisplatin, ifosfamide, etoposide, dexamethasone, L-asparaginase; RCT, Randomized controlled trial; OR, overall response rate; OS, overall survival.

up of 36 months, the cumulative incidence of non-relapse mortality and relapse at 3 years were $30 \%$ and $42 \%$, respectively. The corresponding 3-year PFS and OS were $28 \%$ and $34 \%$, respectively.

American Society for Blood and Marrow Transplantation guidelines recommend allogeneic-HSCT for advanced ENKL after relapse, refractory disease, and in refractory localized disease. ${ }^{18}$

Most studies have been retrospective; therefore, further investigations are needed to explore the most appropriate use of allogeneic-HSCT to treat ENKL.

\section{Therapy Using PD-I or Programmed Cell Death Protein I Ligand (PD-LI) PDLI Expression on ENKL}

The PD1/PD-L1 pathway plays an important part in tumor immunity. PD-L1 is expressed broadly on normal cells, such as dendritic cells, macrophages, activated T cells, and epithelial cells. However, PD-L1 can be expressed widely on solid tumors and lymphomas, and this is associated with a poor prognosis. ${ }^{21,22}$ In terms of ENKL, Kim et al reported PD-L1 expression in ENKL cells in $56.2 \%$ of cases, and in $62 \%$ of total cells including malignant and non-malignant cells. The patients with PD-L1 ${ }^{+}$ENKL were associated with a better 5-year overall survival (OS) prognosis. ${ }^{23}$ Nagato et al found that PD-L1 was expressed not only on ENKL cells but also on tumor-infiltrating macrophages. Moreover, higher expression of soluble PD-L1 has been detected in ENKL patients than that in healthy individuals, and is correlated with a poor prognosis. ${ }^{24}$ Interferon (IFN)- $\gamma$ can induce PD-L1 expression on macrophages and is also secreted by ENKL cells. ${ }^{25,26}$ Therefore, PD-1/PD-L1 may inhibit tumor cells directly and inhibit macrophages indirectly.

\section{Signaling Pathway of PD-I and PD-LI}

PD-1 plays a vital part in the maintenance of peripheral tolerance. ${ }^{27}$ Furthermore, PD-1 and its ligand PD-L1 have important roles in tumor-associated immunosuppression. Tumor antigens can induce PD-1 overexpression by tumorinfiltrating lymphocytes, and PD-L1 expression is a characteristic of malignant cells and various tumor-infiltrating antigen-presenting cells. ${ }^{28}$ PD-1 contains two tyrosine molecules, ITIM (immune receptor tyrosine-based inhibitory motif) and ITSM (immune receptor tyrosine-based switch motif), in its cytoplasmic tail. ${ }^{6}$ In the presence of PDL1, Src homology region 2 domain-containing phosphatase-2 (SHP-2) and SHP1 are recruited to ITSM in PD-1. ${ }^{29}$ With the 
participation of T-cell receptors, PD-L1 can inhibit phosphatidylinositol-4,5-bisphosphate 3-kinase (PI3K) and the downstream activity of the protein kinase B (Akt) signaling pathway. ${ }^{30}$ In addition, PD-L1 can inhibit PKC $\theta$ (Protein kinase $\mathrm{C}$ theta) and RAS-Erk (Ras-extracellular signal-regulated kinase) signaling. ${ }^{18}$ Upon B-cell receptor (BCR) engagement, PD-1 can also inhibit BCR signaling by recruiting SHP-2 to its phosphotyrosine residues, and dephosphorylate key signal transducers of BCR signaling, including kinase (Syk), phospholipase C-gamma 2, PI3K, and vav. ${ }^{31}$

\section{Clinical Treatments and Adverse Events}

Several clinical trials have demonstrated that treatment involving PD-1 blockade as a target in hematological tumors is promising. ${ }^{32}$ Classical Hodgkin's lymphoma is an encouraging target for anti-PD-1 therapy because amplification of the 9p24.1 locus increases PD-L1 expression on Reed-Sternberg cells. ${ }^{33}$ Pembrolizumab and nivolumab are the most common humanized recombinant monoclonal antibodies against PD- $1 .{ }^{34}$ The mechanism is illustrated in Figure 1. Due to the low incidence of ENKL, PD-1/PD-L1 have not been tested in large-scale clinical trials. However, small-scale clinical application has achieved encouraging outcomes. Kwong et al enrolled seven male ENKL patients. The median age was 49 (range, 31-68) years, and all patients had relapsed disease after failure of at least one type of regimen, including L-asparaginase-containing regimens and allogeneic-HSCT.

Patients received pembrolizumab $(2 \mathrm{mg} / \mathrm{kg})$ every 3 weeks except one patient who received pembrolizumab every 2 weeks. Two patients achieved a complete response (CR), three patients achieved clinical and radiological CRs, and two patients achieved a partial response (PR). The five patients who achieved a CR were still in remission after a median follow-up of 6 (range, 2-10) months. Immune-related adverse events were not seen except in one patient who had allogeneic-HSCT previously and suffered grade-2 skin graft-versus-host disease.

Another trial was conducted in seven heavily pretreated ENKL patients. ${ }^{7}$ All patients had received at least two chemotherapy regimens previously, and pembrolizumab $(100 \mathrm{mg})$ was administered every 3 weeks in all patients. After a median of four cycles of treatment (range, 2-18), among the seven patients, two had a CR and two had a PR, and the overall response rate was $57 \%$. PDL1 expressed on lymphoma cells showing $50 \%, 20 \%, 30 \%, 70 \%$, and $30 \%$ of five patients, respectively. PD-L1 expression was not detected in one patient and was negative in one patient. The main treatment-related adverse events were pneumonitis, laboratory abnormalities, diarrhea, fever, and thrombocytopenia. One patient developed grade 3 thrombocytopenia and the thrombocyte count recovered after treating with thrombocyte transfusion and recombinant human thrombopoietin. Other patients suffered from grade 3 pneumonitis. However, these were tolerable and could be managed safely.

Nivolumab treatment was evaluated in three patients with relapsed ENKL who were refractory to treatment. ${ }^{8}$ Patient $\# 1$ and \#3 were given two doses of nivolumab (total of $80 \mathrm{mg}$ ) and responses were obtained. Because of poor conditions,

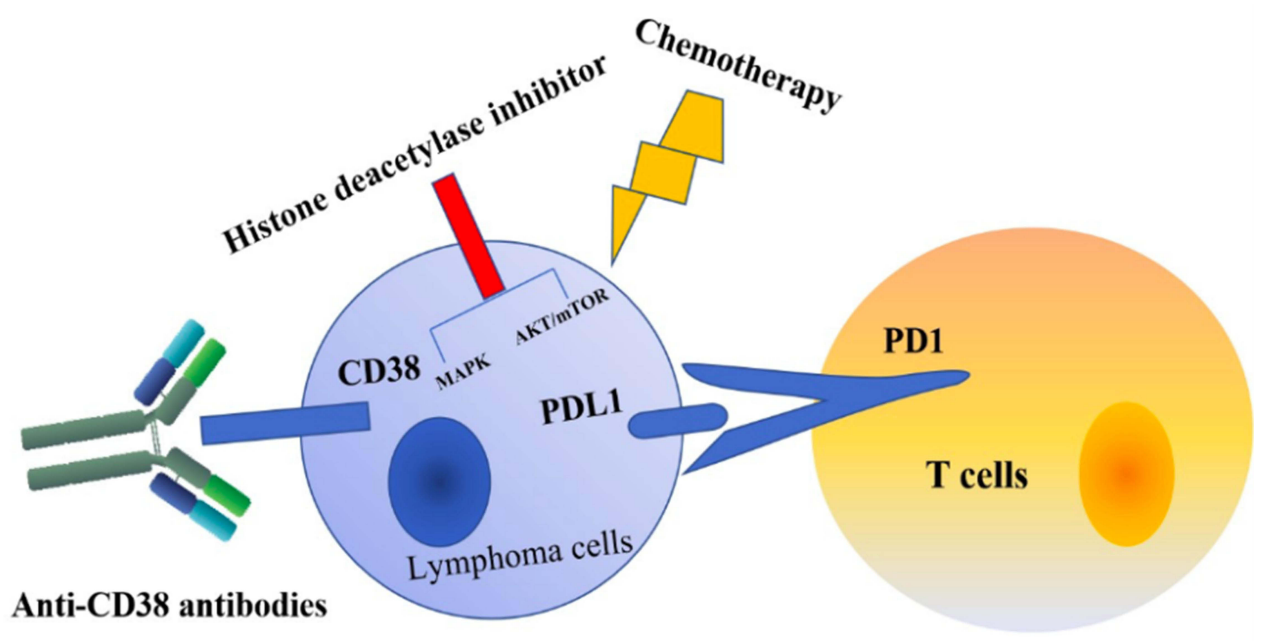

Figure I Possible T-cell lymphoma treatments. 
two patients died from infections. Patient \#2 was given nivolumab $(160 \mathrm{mg})$ and obtained a $\mathrm{CR}$ and radiological response. This patient received $>240 \mathrm{mg}$ and achieved a CR. Patient $\# 3$ had a high tumor load and developed tumor lysis syndrome and grade 1 cytokine release syndrome after receiving nivolumab. Toxicity was not observed in the other two patients. Other promising studies investigating the role of PD-1/PD-L1 therapy in ENKL are ongoing.

\section{Histone Deacetylase Inhibitors (HDACIs)}

HDACIs have attracted considerable attention because they can be used to treat various types of tumor. They have been approved for treatment of relapsed and refractory peripheral T-cell lymphoma. They can modulate chromatin remodeling and maintain an acetylation balance among DNA damage-related proteins.

Zhou et al investigated the oral HDACI chidamide, which showed antitumor effects by inhibiting the Akt/mammalian target of rapamycin and mitogen-activated protein kinase signaling pathways in natural killer T-cell lymphoma cell lines. Moreover, chidamide can also activate the ATM-Chk2-p53-p21 signaling pathway in vitro. ${ }^{35}$

Five patients were enrolled in an open-label, prospective pilot study to evaluate the role of romidepsin in the treatment of patients with ENKL. However, three patients had fever and increased levels of liver enzymes and bilirubin after the first administration of romidepsin. Additionally, EBV DNA titers in blood from these patients were increased. The authors considered EBV reactivation and discontinued patient enrollment. ${ }^{36}$ Therefore, the current view is that single use of HDACIs is not recommended.

\section{Cluster of Differentiation (CD)38 Monoclonal Antibody}

There have been sporadic reports that CD38 monoclonal antibody can be used in relapsed and refractory ENKL. In a retrospective study, CD38 expression in 94 ENKL patients revealed that half of patients had high expression of CD38, thereby indicating the potential role of CD38 as a therapy target for ENKL. ${ }^{37}$

Shoag et al reported a patient with relapsed ENKL who underwent L-asparaginase-based chemotherapy and allogeneic-HSCT. They gave the patient daratumumab $(16 \mathrm{mg} / \mathrm{kg}$ bodyweight per week). After 6 weeks of treatment, a CR at 21-week follow-up was documented. ${ }^{38}$

\section{Chimeric Antigen Receptor-T Cell (CAR-T) Therapy}

The CAR-T therapy is a novel immunotherapy and two products have been approved for acute lymphoblastic leukemia (ALL) and adult diffuse large B-cell lymphoma subtypes (DLBCL). ${ }^{39} \mathrm{CARs}$ are artificial fusion proteins which include an antigen-recognition domain and T-cell signaling domains. ${ }^{40}$ The antigen-recognition domain is called single-chain variable

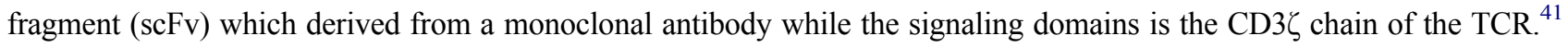

CAR-T therapy has shown significant results in some refractory hematologic malignancies. ${ }^{42}$ Moreover, It is also being investigated in some solid tumors such as glioblastoma, HER2-Positive Sarcoma and so on. ${ }^{43,44}$ Up to now, reports using CAR-T therapy for ENKL are lacking, but treatment of ENKL utilizing specific antigens of ENKL may represent a new approach.

\section{Conclusions}

The possible treatments developed to treat ENKL are shown in Figure 1. ENKL is a peculiar form of lymphoma associated with EBV infection. Because of frequent P-glycoprotein expression, anthracycline-based regimens are not efficacious. L-asparaginase-containing chemotherapy, such as the SMILE protocol, may improve the prognoses of patients with ENKL. Single or combined use of PD-1/PD-L1 inhibitors have elicited encouraging outcomes. The curative effect of auto-HSCT in ENKL is not clear, but allogeneic-HSCT can be considered in advanced ENKL after relapse or in refractory ENKL. Use of HDACIs and CD38 monoclonal antibody has shown promise. More clinical trials are needed for immunotherapy of ENKL.

\section{Funding}

This research was supported financially by National Natural Science Foundation of China (81971172), Zhejiang Natural Science Foundation (LY19H160037, LY18C09004), Medical and Health Science and Technology Project of Zhejiang 
Province (2017KY228, 2018KY003, 2018KY816), The Key Research and Development Program of Zhejiang Province (WKJ-ZJ-1914), Zhejiang Public Welfare Technology Research Program (LGF18H090009) and Health Commission of Zhejiang Province (2018KY020).

\section{Disclosure}

The authors declared that they have no conflicts of interest to this work.

\section{References}

1. Yamaguchi M, Kita K, Miwa H. Frequent expression of P-glycoprotein/MDR1 by nasal T-cell lymphoma cells. Cancer. 1995;76(11):2351-2356. doi:10.1002/1097-0142(19951201)76:11<2351::AID-CNCR2820761125>3.0.CO;2-1

2. Swerdlow SH, Campo E, Harris NL, et al. WHO Classification of Tumours of Haematopoietic and Lymphoid Tissues (Revised 4th Edition). Lyon: IARC; 2017.

3. Kim WY, Montes-Mojarro IA, Fend F, Quintanilla-Martinez L. Epstein-Barr virus-associated T and NK-cell lymphoproliferative diseases. Front Pediatr. 2019;7:71. doi:10.3389/fped.2019.00071

4. Yamaguchi M, Suzuki R, Oguchi M. Advances in the treatment of extranodal NK/T-cell lymphoma, nasal type. Blood. 2018;131(23):2528-2540. doi:10.1182/blood-2017-12-791418

5. Yamaguchi M, Kwong YL, Kim WS, et al. Phase II study of SMILE chemotherapy for newly diagnosed stage IV, relapsed or refractory extranodal NK/T-cell lymphoma, nasal type: the NK-cell tumor study group (NKTSG) study. J Clin Oncol. 2011;29(33):4410-4416. doi:10.1200/ JCO.2011.35.6287

6. Jaccard A, Gachard N, Marin B, et al. Efficacy of L-asparaginase with methotrexate and dexamethasone (AspaMetDex regimen) in patients with refractory or relapsing extranodal NK/T-cell lymphoma, a Phase 2 study. Blood. 2011;117(6):1834-1839. doi:10.1182/blood-2010-09307454

7. Li X, Cheng Y, Zhang M, et al. Activity of pembrolizumab in relapsed/refractory NK/T-cell lymphoma. J Hematol Oncol. 2018;11(1):15. doi:10.1186/s13045-018-0559-7

8. Chan TSY, Li J, Loong F, et al. PD1 blockade with low-dose nivolumab in NK/T cell lymphoma failing L-asparaginase: efficacy and safety. Ann Hematol. 2018;97(1):193-196. doi:10.1007/s00277-017-3127-2

9. Isobe K, Uno T, Tamaru J, et al. Extranodal natural killer/T-cell lymphoma, nasal type: the significance of radiotherapeutic parameters. Cancer. 2006;106(3):609-615. doi:10.1002/cncr.21656

10. Yang Y, Zhu Y, Cao JZ, et al. Risk-adapted therapy for early-stage extranodal nasal-type NK/T-cell lymphoma: analysis from a multicenter study. Blood. 2015;126(12):1424-1432; quiz 1517. doi:10.1182/blood-2015-04-639336

11. Kwong YL, Kim WS, Lim ST, et al. SMILE for natural killer/T-cell lymphoma: analysis of safety and efficacy from the Asia Lymphoma Study Group. Blood. 2012;120(15):2973-2980. doi:10.1182/blood-2012-05-431460

12. Qi S, Yahalom J, Hsu M, et al. Encouraging experience in the treatment of nasal type extra-nodal NK/T-cell lymphoma in a non-Asian population. Leuk Lymphoma. 2016;57(11):2575-2583. doi:10.1080/10428194.2016.1180689

13. NCCN guidelines T-cell lymphomas version I; 2021. Available from: https://www.nccn.org/professionals/physician_gls/pdf/t-cell.pdf. Accessed January 18, 2022.

14. Wang JH, Wang L, Liu CC, et al. Efficacy of combined gemcitabine, oxaliplatin and pegaspargase (P-gemox regimen) in patients with newly diagnosed advanced-stage or relapsed/refractory extranodal NK/T-cell lymphoma. Oncotarget. 2016;7(20):29092-29101. doi:10.18632/ oncotarget. 8647

15. Li X, Cui Y, Sun Z, et al. DDGP versus SMILE in newly diagnosed advanced natural killer/T-cell lymphoma: a randomized controlled, multicenter, open-label study in China. Clin Cancer Res. 2016;22(21):5223-5228. doi:10.1158/1078-0432.CCR-16-0153

16. Lee J, Au WY, Park MJ, et al. Autologous hematopoietic stem-cell transplantation in extranodal natural killer/T cell lymphoma: a multinational, multicenter, matched controlled study. Biol Blood Marrow Transplant. 2008;14(12):1356-1364. doi:10.1016/j.bbmt.2008.09.014

17. Yamaguchi M, Suzuki R, Oguchi M, et al. Treatments and outcomes of patients with extranodal natural killer/T-cell lymphoma diagnosed between 2000 and 2013: a cooperative study in Japan. J Clin Oncol. 2017;35(1):32-39. doi:10.1200/JCO.2016.68.1619

18. Yhim HY, Kim JS, Mun YC, et al. Clinical outcomes and prognostic factors of up-front autologous stem cell transplantation in patients with extranodal natural killer/T cell lymphoma. Biol Blood Marrow Transplant. 2015;21(9):1597-1604. doi:10.1016/j.bbmt.2015.05.003

19. Tse E, Chan TS, Koh LP, et al. Allogeneic haematopoietic SCT for natural killer/T-cell lymphoma: a multicentre analysis from the Asia Lymphoma Study Group. Bone Marrow Transplant. 2014;49(7):902-906. doi:10.1038/bmt.2014.65

20. Kanate AS, DiGilio A, Ahn KW, et al. Allogeneic haematopoietic cell transplantation for extranodal natural killer/T-cell lymphoma, nasal type: a CIBMTR analysis. Br J Haematol. 2018;182(6):916-920. doi:10.1111/bjh.14879

21. Brahmer JR, Drake CG, Wollner I, et al. Phase I study of single-agent anti-programmed death-1 (MDX-1106) in refractory solid tumors: safety, clinical activity, pharmacodynamics, and immunologic correlates. J Clin Oncol. 2010;28(19):3167-3175. doi:10.1200/JCO.2009.26.7609

22. Kiyasu J, Miyoshi H, Hirata A, et al. Expression of programmed cell death ligand 1 is associated with poor overall survival in patients with diffuse large B-cell lymphoma. Blood. 2015;126(19):2193-2201. doi:10.1182/blood-2015-02-629600

23. Kim WY, Jung HY, Nam SJ, et al. Expression of programmed cell death ligand 1 (PDL1) in advanced stage EBV-associated extranodal NK/T cell lymphoma is associated with better prognosis. Virchows Arch. 2016;469(5):581-590. doi:10.1007/s00428-016-2011-0

24. Nagato T, Ohkuri T, Ohara K, et al. Programmed death-ligand 1 and its soluble form are highly expressed in nasal natural killer/T-cell lymphoma: a potential rationale for immunotherapy. Cancer Immunol Immunother. 2017;66(7):877-890. doi:10.1007/s00262-017-1987-x

25. Zou W, Chen L. Inhibitory B7-family molecules in the tumour microenvironment. Nat Rev Immunol. 2008;8(6):467-477. doi:10.1038/nri2326

26. Takahara M, Kis LL, Nagy N, et al. Concomitant increase of LMP1 and CD25 (IL-2-receptor alpha) expression induced by IL-10 in the EBV-positive NK lines SNK6 and KAI3. Int J Cancer. 2006;119(12):2775-2783. doi:10.1002/ijc.22139 
27. Okazaki T, Iwai Y, Honjo T. New regulatory co-receptors: inducible co-stimulator and PD-1. Curr Opin Immunol. 2002;14(6):779. doi:10.1016/ S0952-7915(02)00398-9

28. Xia Y, Jeffrey Medeiros L, Young KH. Signaling pathway and dysregulation of PD1 and its ligands in lymphoid malignancies. Biochim Biophys Acta. 2016;1865(1):58-71. doi:10.1016/j.bbcan.2015.09.002

29. Chemnitz JM, Parry RV, Nichols KE, et al. SHP-1 and SHP-2 associate with immunoreceptor tyrosine-based switch motif of programmed death 1 upon primary human $\mathrm{T}$ cell stimulation, but only receptor ligation prevents T cell activation. J Immunol. 2004;173(2):945-954. doi:10.4049/ jimmunol.173.2.945

30. Parry RV, Chemnitz JM, Frauwirth KA, et al. CTLA-4 and PD-1 receptors inhibit T-cell activation by distinct mechanisms. Mol Cell Biol. 2005;25 (21):9543-9553. doi:10.1128/MCB.25.21.9543-9553.2005

31. Okazaki T, Maeda A, Nishimura H, Kurosaki T, Honjo TPD-1. immunoreceptor inhibits B cell receptor-mediated signaling by recruiting src homology 2-domain-containing tyrosine phosphatase 2 to phosphotyrosine. Proc Natl Acad Sci USA. 2001;98(24):13866-13871. doi:10.1073/ pnas. 231486598

32. Ok CY, Young KH. Checkpoint inhibitors in hematological malignancies. J Hematol Oncol. 2017;10(1):103. doi:10.1186/s13045-017-0474-3

33. Green MR, Monti S, Rodig SJ, et al. Integrative analysis reveals selective 9p24.1 amplification, increased PD-1 ligand expression, and further induction via JAK2 in nodular sclerosing Hodgkin lymphoma and primary mediastinal large B-cell lymphoma. Blood. $2010 ; 116(17): 3268-3277$. doi:10.1182/blood-2010-05-282780

34. Kwong YL, Chan TSY, Tan D, et al. PD1 blockade with pembrolizumab is highly effective in relapsed or refractory NK/T-cell lymphoma failing 1-asparaginase. Blood. 2017;129(17):2437-2442. doi:10.1182/blood-2016-12-756841

35. Zhou J, Zhang C, Sui X, et al. Histone deacetylase inhibitor chidamide induces growth inhibition and apoptosis in NK/T lymphoma cells through ATM-Chk2-p53-p21 signalling pathway. Invest New Drugs. 2018;36(4):571-580. doi:10.1007/s10637-017-0552-y

36. Kim SJ, Kim JH, Ki CS, et al. Epstein-Barr virus reactivation in extranodal natural killer/T-cell lymphoma patients: a previously unrecognized serious adverse event in a pilot study with romidepsin. Ann Oncol. 2016;27(3):508-513. doi:10.1093/annonc/mdv596

37. Wang L, Wang H, Li PF, et al. CD38 expression predicts poor prognosis and might be a potential therapy target in extranodal NK/T cell lymphoma, nasal type. Ann Hematol. 2015;94(8):1381-1388. doi:10.1007/s00277-015-2359-2

38. Hari P, Raj RV, Olteanu H. Targeting CD38 in refractory extranodal natural killer cell-T-cell lymphoma. $N$ Engl J Med. $2016 ; 375(15): 1501-1502$. doi:10.1056/NEJMc1605684

39. Brudno JN, Kochenderfer JN. Recent advances in CAR T-cell toxicity: mechanisms, manifestations and management. Blood Rev. 2019;34:45-55. doi:10.1016/j.blre.2018.11.002

40. Sadelain M, Brentjens R, Rivière I. The basic principles of chimeric antigen receptor design. Cancer Discov. 2013;3(4):388-398. doi:10.1158/21598290.CD-12-0548

41. Rouce RH, Sharma S, Huynh M, Heslop HE. Recent advances in T-cell immunotherapy for haematological malignancies. Br J Haematol. $2017 ; 176$ (5):688-704. doi:10.1111/bjh.14470

42. Grupp SA, Kalos M, Barrett D, et al. Chimeric antigen receptor-modified T cells for acute lymphoid leukemia. $N$ Engl $J$ Med. 2013;368 (16):1509-1518. doi:10.1056/NEJMoa1215134

43. Brown CE, Alizadeh D, Starr R, et al. Regression of Glioblastoma after Chimeric Antigen Receptor T-Cell Therapy. $N$ Engl $J$ Med. 2016;375 (26):2561-2569. doi:10.1056/NEJMoa1610497

44. Ahmed N, Brawley VS, Hegde M, et al. Human Epidermal Growth Factor Receptor 2 (HER2) -specific chimeric antigen receptor-modified T cells for the immunotherapy of HER2-positive sarcoma. J Clin Oncol. 2015;33(15):1688-1696. doi:10.1200/JCO.2014.58.0225

45. Dong LH, Zhang LJ, Wang WJ, et al. Sequential DICE combined with 1-asparaginase chemotherapy followed by involved field radiation in newly diagnosed, stage IE to IIE, nasal and extranodal NK/T-cell lymphoma. Leuk Lymphoma. 2016;57(7):1600-1606. doi:10.3109/ 10428194.2015 .110841

Cancer Management and Research is an international, peer-reviewed open access journal focusing on cancer research and the optimal use of preventative and integrated treatment interventions to achieve improved outcomes, enhanced survival and quality of life for the cancer patient. The manuscript management system is completely online and includes a very quick and fair peer-review system, which is all easy to use. Visit http://www.dovepress.com/testimonials.php to read real quotes from published authors.

Submit your manuscript here: https://www.dovepress.com/cancer-management-and-research-journal 